\title{
Nanoadsorbents for the preconcentration of some toxic substances: a minireview
}

\author{
Anupreet Kaur *, Shivender Singh Saini ** \\ Department of Chemistry, Punjabi University, Patiala - 147002, Punjab, India \\ *,**E-mail address: anu_chem06@yahoo.co.in , shiv_algal2007@yahoo.com
}

\begin{abstract}
The development of new sorbents and their application in preconcentration methods for determination of trace analytes is subject of great interest. Sample pretreatment methods, such as separation / preconcentration prior to the determination of metal ions have developed rapidly due to the increasing need for accurate and precise measurements at extremely low levels of ions in diverse matrices. This review summarizes and discusses several analytical methods involving the preparation and use of new solid phase extractant. A literature survey of the last ten years offering a critical review of these new sorbents available for use in trace analyte enrichment is provided.
\end{abstract}

Keywords: Solid phase extraction; preconcentration; trace analytes

\section{INTRODUCTION}

Separation and preconcentration techniques are of great importance owing to the limited sensitivity of modern instrumental methods for trace analysis. Pre-treatment of an aqueous sample by different sorption technique not only increases the ion concentration to a detectable level but also eliminates matrix effects. The use of chelating sorbents can provide a concentration factor up to several hundred folds, better separation of interferent ions and high efficiency. The general trend of modern analytical chemistry is towards the elaboration of simple, ecologically safe, sensitive, and selective methods for the determination of trace components combining previous concentration methods and further determination by physical or physico-chemical methods. Pollutant quantification at low concentration levels comprises one of the most considered targets in analytical chemistry.

Enrichment is attained by the use of various preconcentration techniques based on physical, physio-chemical and chemical principle. The techniques generally employed in analytical chemistry are liquid-liquid extraction, electrochemical method, ion-exchange, coprecipitation and solid phase extraction. Electrochemical deposition used for the preconcentration of different pollutants by applying the laws of electrolysis in which cationic species are deposited on the electrode surface. The only disadvantage of this method is that limitation related to $\mathrm{pH}$ control. This control is necessary because in the acid medium, hydrogen ions are reduced to hydrogen gas on the work electrode surface. The hydrogen gas generation occurs when more negative potentials are applied. The reduction of electrode lifetimes is also observed at higher acidity conditions. In coprecipitation or precipitation is characterized by the formation of insoluble compounds. The coprecipitation is adopted when 
direct precipitation can not separate the desired metallic species due to its low concentration in solution. The coprecipitation phenomenon can be associated with metal adsorption on the precipitate surface or due to metal incorporation onto the precipitate structures. The coprecipitation occurs by the formation of an insoluble compound containing some metallic species. Thus, there is a natural limitation according to this phenomenon, because the metal used for this purpose cannot be determined. The separation and preconcentration of metal ions and organic pollutants, after the formation of sparingly water-soluble complex, based on cloud point extraction have been largely employed in analytical chemistry. Current research in this field has focused on the development of new surfactant phase separations that surpassed the limitations associated with non-ionic surfactants. The search for alternatives to traditional organic solvents in liquid-liquid extraction has fostered the use of more environmentally friendly liquids. Cloud point extraction that is the temperatureinduced phase separation of nonionic surfactants, continues as one of the leading techniques for the preconcentration of metal ions. But application of cloud point extraction to the extraction of organic pollutants is less straightforward because of the coelution problems originated by non-ionic surfactants which are commercially available as a mixture of homologues and isomers.

But according to Hitherto, liquid-liquid extraction is among the most often used method for the various preconcentration or separation techniques in view of its simplicity, rapidity, ready adaptability and easier recovery of analyte, There are, however physical difficulties associated with the use of solvent extraction for enrichment of large number of samples and /or requires vigorous agitation to ensure complete partition of the analyte between two immiscible phases, and this can be achieved only by the application of significant human or mechanical effort. In addition, there are increasing environmental and cost pressures to replace, or at the very least reduce, the volume of solvents employed in analytical procedures. Current trends in preconcentration focus on the development of faster, safer and more environment friendly extraction techniques. Solid phase extraction continues to be the leading technique for the extraction of pollutants in aquatic systems; recent developments in this field are mainly related to the use of new sorbents. Solid phase extraction (SPE) has emerged as a powerful tool for separation/ enrichment of inorganics, organics and biomolecules. The basic principle of SPE is transfer of analytes from aqueous phase to active sites of adjacent solid phase. Recently, solid-phase extraction technique for preconcentration of heavy metal ions has become very popular, compared with traditional solvent extraction techniques and has almost replaced liquid-liquid extraction techniques because of several advantages. The fast, simple and direct sample application in very small size (micro liter volume) without any sample loss.

(1) Higher preconcentration factor.

(2) The ability of combination with different modern analytical techniques.

(3) Time and cost saving.

(4) There is no use of organic solvents which are flammable, toxic and even some of them carcinogenic.

(5) Absence of emulsion.

(6) Rapid phase separation.

(7) Stability and re-usability of solid phase.

(8) Isolate analytes from large volumes of sample with minimal or zero evaporation losses. 
An analytical chemist is always in search of cheaper, quicker, more sensitive, more reliable, precise methods of analysis. To achieve such a goal many properties of the materials are studied. Nanotechnology meets many of the conditions mentioned above and is very economic. So, analytical nanotechnology is an important tool for preconcentration and separation of pollutants at low levels. The role of analytical science in nanoscience and nanotechnology has been clearly delimited within the almost universally accepted limits of the "nanoscale" (from 1 to $100 \mathrm{~nm}$ ). The "nanoscale" concept has introduced a new scenario where physicochemical principles, laws and properties are quite different from those of the macro and micro worlds. The most extensively explored area of analytical nanotechnology is to exploit the excellent properties of nanoparticles to improve well-established analytical methods or to develop new methods for analytes or matrices. In addition to the typical advantages of nanoparticles, their use should lead to improved selectivity, sensitivity, rapidity, miniaturizability or portability of the analytical system. Nano-materials, with a new series of different physical and chemical properties superior to the traditional materials, is the basis of nanotechnology. Nanoparticles can be used for purposes such as sample treatment, instrumental separation of analytes, or even detection. In combination with the large variety of nanoparticles available, this provides a wide range of potential applications. Sample pretreatment methods, such as preconcentration and/or separation prior to the determination of analytes (metal ions) have developed rapidly due to the increasing need for accurate and precise measurements at extremely low levels of ions in diverse matrices. Among the separation/preconcentration methods, solid-phase extraction (SPE) has become the most frequently used technique for trace analysis. Nanoparticles show different physical and chemical properties from larger particles of the same materials.

The reasons for changes in reactivity at the nanoscale can be rationalized through four interrelated mechanisms: (1) as nanoparticles get smaller and smaller, the proportion of atoms at the surface or near-surface regions increases dramatically, often causing an increasing reactive surface area depending on the change in the distribution of surface edges, steps, kinks and terraces (2) as a result, the surface free energy of the particle will change as function of particle size, thus influencing the thermodynamics of chemical reactivity (3) atomic structure variations occur, in terms of change in bond lengths, bond angles and vacancies and other defects near and on surface (4) size-quantization effects modify the electronic structure of material as the band structure, begins to resemble discrete energy states of small molecule.

Hybrid materials offer the opportunity to combine the desirable properties of organic compounds. The mild synthetic conditions offered by Sol-gel process allow the mixing of inorganic-organic components at the nanometric scale. Since then, the study of so-called functional hybrid nanocomposities became a mushrooming field of investigation yielding innovative advanced materials with high added value. The major driving forces behind the intense activities in this area are the new and different properties of the nanocomposites which the traditional macroscale composites and conventional materials do not have. The traditional composite materials have macroscale domain size of millimeter and even micrometer scale, most of the inorganic-organic hybrid materials are nanoscopic with the physical constraint of several nanometer typically $1-100 \mathrm{~nm}$ as minimum size of components.

These hybrid nanoparticles being at the interface of inorganic and organic realms are highly versatile which offer a wide range possibility to elaborate tailor made materials in terms of processing, their chemical and physical properties. The most obvious advantage of inorganic-organic hybrids is that they can favorably combine the often dissimilar properties 
of inorganic and organic components in one material. Because of many possible combinations of components, this field is very creative, since it provides opportunity to invent an almost unlimited set of new materials with a large spectrum of known and as yet unknown properties. nanometer-size powder materials were frequently used for the separation and enrichment of trace elements. Additionally, the coating of complexing reagents onto nanomaterials increase the number of binding sites and enable to interact with metal ions and changes the binding sites in order to enhance the uptake of metal ions. Recent advancements suggest that many of issues involving water quality could be resolved or greatly ameliorated using nanoparticles. Innovative use of nanoparticles for treatment of industrial wastewater is another potentially useful application. Many industries generate large amounts of waste water. Removal of contaminants and recycling of the purified water would provide significant reduction in cost, time and energy to the industry and result in the improved environmental stewardship. Aquifer and groundwater remediation are also critical issue, becoming more important as water supplies steadily decrease and demand continues to increase. Most of remediation technologies available today, while effective, very often are costly and time consuming.

The ability to remove toxic components from subsurface and other environments that are very difficult to access in situ. Nanoparticles in analytical chemistry is the most extensively explored areas of nanotechnology. The objective is to exploit the excellent properties of nanoparticles to improve analytical methods or to develop new ones for the analytes or matrices. Nanoparticles have two key properties that make them particularly attractive sorbent. In addition to the typical advantages of nanoparticles, their use should lead to improved selectivity, sensitivity, rapidity, miniaturizability or portability of the analytical system. Nanoparticles can be incorporated or used in analytical methods either as such or chemically grafted. In the latter case, nanoparticles can be chemically bonded to a surface or functionalized with other organic or inorganic compounds in order to increase their sorption capacity. Chemically unmodified nanoparticles can be used as raw randomized materials or as self assembled raw materials. Nanoparticles can be used for purposes such as sample treatment, instrumental separation of analytes, or detection. In combination with the large variety of nanoparticles available, this provides a wide range of potential applications.

The nanoparticles most widely used in analytical sciences at present include (a) silica nanoparticles (b) carbon nanoparticles (mainly fullerenes and carbon nanotubes) (c) metallic nanoparticles (d) supramolecular aggregates. Nanoparticles can also be functionalized with various chemical groups to increase their affinity towards target analytes. The unique properties of nanoparticles have been used to develop high capacity and selective sorbents for metal ions and pollutants. Due to these reasons, the nanoparticles are synthesized and designed to act as either extractants or reaction media for pollutants or scaffolds and delivery vehicles for bioactive compounds; thus providing unprecedented opportunities to develop more efficient and cost effective water purification processes and systems. Consequently, nanometer material can selectively adsorb metal ions and have a very high adsorption capacity.

This review briefly deals with recent advances and applications of nanotechnology for removal of environmental pollutants. Under the nanotechnology umbrella, a number of new procedures for producing nanomaterials ultimately used for treatment of waste water are presented. Research advances for the use of metals, bimetallic nanoparticles, mixed oxides, carbon nanomaterials in remediation of environment are also reviewed. 
Metal-Oxide Nanoparticles

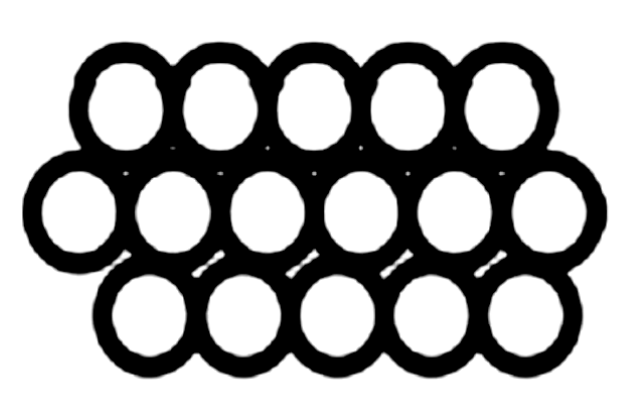

Zeolites

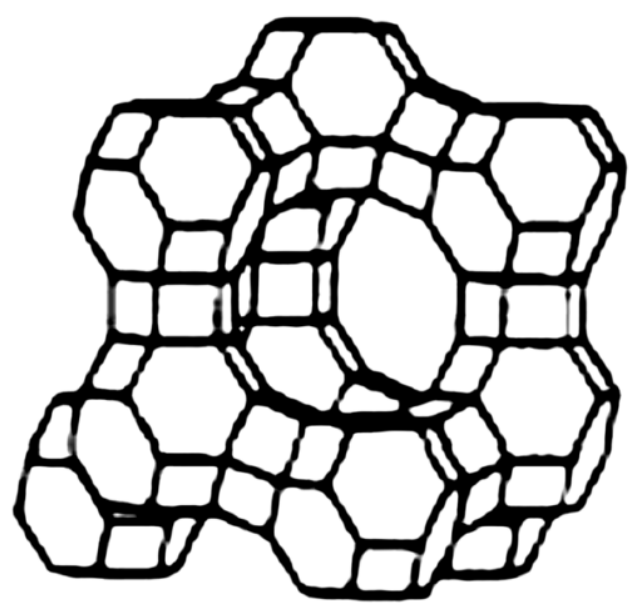

Carbon Nanotubes
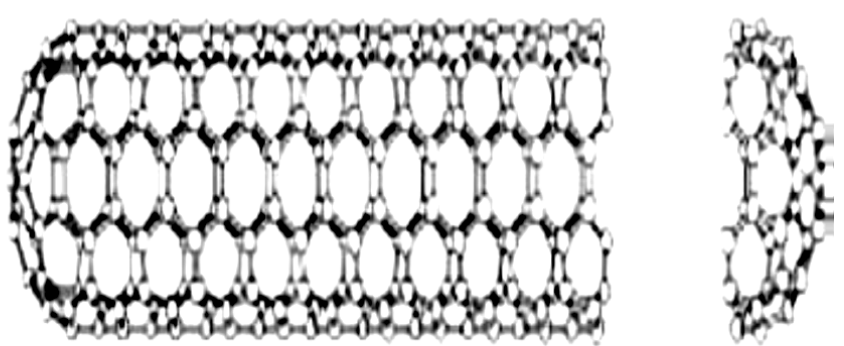

Figure 1. Selected nanomaterials currently being evaluated as functional materials for water purification.

\section{APPLICATION OF NANOPARTICLES FOR THE REMOVAL OF VARIOUS POLLUTANTS}

The selective sorption of certain elements based on the stability of complexes formed with functional groups of sorbents, has led to the use of these materials for selective enrichments and separation of inorganic ions from different natural and industrial sources. According to researchers at the Pacific North Laboratory (PNL), a unique chemically modified nanoporous ceramics can remove contaminants from waste streams faster and at a significantly lower cost than conventional techniques such as ion-exchange resins and activated carbon filters. This nanosponge could be used in a wide range of environmental applications, including drinking-water purification, waste water treatment, site remediation and waste stabilization.

Nanometer-sized metal oxides, such as $\mathrm{Al}_{2} \mathrm{O}_{3}, \mathrm{TiO}_{2}, \mathrm{ZrO}_{2}, \mathrm{CeO}_{2}$, and $\mathrm{SiO}_{2}$ exhibit intrinsic surface reactivity and high surface areas and can strongly chemisorb several substances. In recent years Advanced Oxidation Processes (AOPs) using titanium dioxide $\left(\mathrm{TiO}_{2}\right)$ have been effectively used to detoxify recalcitrant pollutants present in industrial wastewater. $\mathrm{TiO}_{2}$ have singular characteristics that made it an extremely attractive photocatalyst, high photochemical reactivity, high photocatalytic activity, low cost, stability 
in aquatic systems and low environmental toxicity. The general detailed mechanism of dye degradation upon irradiation is described below:

$$
\begin{aligned}
& \text { Dye }+h v \rightarrow \text { Dye* }^{*} \\
& \text { Dye } *+\mathrm{TiO}_{2} \rightarrow \text { Dye } \bullet++\mathrm{TiO}_{2}(\mathrm{e}) \\
& \mathrm{TiO}_{2}(\mathrm{e})+\mathrm{O}_{2} \rightarrow \mathrm{TiO}_{2}+\mathrm{O}_{2}^{\cdot-} \\
& \mathrm{O}_{2}^{\cdot-}+\mathrm{TiO}_{2}(\mathrm{e})+2 \mathrm{H}^{+} \rightarrow \mathrm{H}_{2} \mathrm{O}_{2} \\
& \mathrm{H}_{2} \mathrm{O}_{2}+\mathrm{TiO}_{2}(\mathrm{e}) \rightarrow \cdot \mathrm{OH}+\mathrm{OH}^{-} \\
& \text {Dye }{ }^{+}+\mathrm{O}_{2}\left(\text { or } \mathrm{O}_{2}{ }^{--} \text {or } \bullet \mathrm{OH}\right) \rightarrow \text { peroxylated or hydroxylated intermediates } \\
& \rightarrow \text { degraded or mineralized products (6) }
\end{aligned}
$$

A $\mathrm{TiO}_{2}$ photocatalyst oxidizes $\mathrm{NO}$ into $\mathrm{NO}_{2}$ and eventually into $\mathrm{NO}_{3}{ }^{-}$, hence removing NO from the air . Nano-gold supported on $\mathrm{TiO}_{2}$ coated glass fiber for removing toxic $\mathrm{CO}$ gas from air. Outstanding catalytic activities of nano-gold for oxidizing $\mathrm{CO}$ at low temperature, various reactions over nano-gold catalysts have been studied. These include $\mathrm{CO}$ oxidation, preferential oxidation of $\mathrm{CO}$ in the presence of excess hydrogen (PROX), water gas shift reaction (WGSR), hydrogenation and oxidation.

Gold nanoparticles (AuNPs), one of the wide variety of core materials available, coupled with tunable surface properties in the form of inorganic or inorganic-organic hybrid have been reported as an excellent platform for a broad range of analytical methods. The modification of the Au surface with appropriate chemical species can improve the separation and preconcentration efficiency, analytical selectivity, and method reliability. Because of their high surface-to-volume ratio, easy surface modification, and simple synthesis methods, gold nanoparticles (AuNPs) are becoming an attractive material as an alternative to conventional solvent extraction and solid phase extraction.

Zero-valent iron nanoparticles are highly reactive and react rapidly with surrounding media in the subsurface. A significant loss of reactivity can occur before the particles are able to reach the target contaminant. In addition, zero-valent iron nanoparticles tend to flocculate when added to water, resulting in a reduction in effective surface area of the metal. Therefore, the effectiveness of a remediation depends on the accessibility of the contaminants to the nanoparticles; and the maximum efficiency of remediation will be achieved only if the metal nanoparticles can effectively migrate without oxidation to the contaminant or the water/ contaminant interface. To overcome such difficulties, a commonly used strategy is to incorporate iron nanoparticles within support materials, such as polymers, porous carbon, and polyelectrolytes.

Silica nanoparticles is a promising materials as a solid phase extractant because of its large surface area, high adsorption capacity, low temperature modification, less degree of unsaturation and low electrophilicity. Chemical modification of nanoparticles by silylation procedure using different silylating agents such as 3-aminopropyltriethoxysilane, 3chloropropyltriethoxysilane and 3-mercaptopropyltriethoxysilane provides immobility, mechanical stability and water insolubility. N-[3-(trimethoxysilyl)propyl]ethylenediammine modified $\mathrm{SiO}_{2}$ nanoparticles have been used for the preconcentration of some toxic heavy metal ions such as $\mathrm{Hg}(\mathrm{II}), \mathrm{Cu}(\mathrm{II}), \mathrm{Zn}(\mathrm{II})$. Modified silica nanoparticles have also been used 
for the preconcentration of drugs and also pesticides. Silylation of silica nanoparticles followed by their chemical modification using 4-(2-pyridylazo)-resorcinol and these modified $\mathrm{SiO}_{2}$ nanoparticles have been used for the selective preconcentration of $\mathrm{Hg}(\mathrm{II})$. $\mathrm{SiO}_{2}$ nanoparticles also modified with acetylsalicylic acid, p-dimethylaminobenzaldehyde and 5-sulfonylsalicylic acid have been used for the preconcentration of $\mathrm{Cr}$ (III), $\mathrm{Fe}(\mathrm{III})$, $\mathrm{Pb}(\mathrm{II})$ and $\mathrm{Cu}(\mathrm{II})$.

Modified $\mathrm{SiO}_{2}$ nanoparticles with PAN, DHAQ and RATP had also been used for the preconcentration of toxic metal ions such $\mathrm{Cd}(\mathrm{II}), \mathrm{Pb}(\mathrm{II}), \mathrm{Sb}(\mathrm{III}), \mathrm{Cu}(\mathrm{II}), \mathrm{Co}(\mathrm{II})$ and also zineb and maneb in environmental samples, food samples studied.

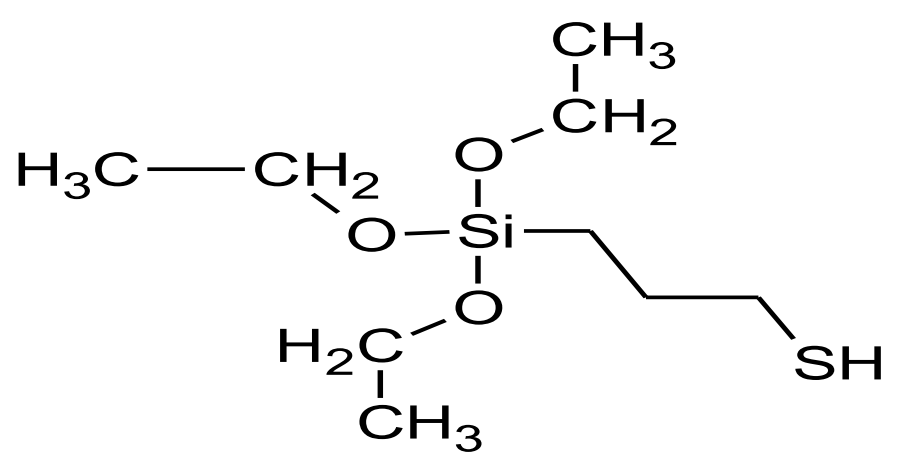

$\mathrm{SiO}_{2}$-3-aminopropyltriethoxysilane nanoparticle<smiles>CCO[Si](CCCS)(OCC)OCC</smiles>

$\mathrm{SiO}_{2}$-3-mercaptopropyltriethoxysilane nanoparticle 


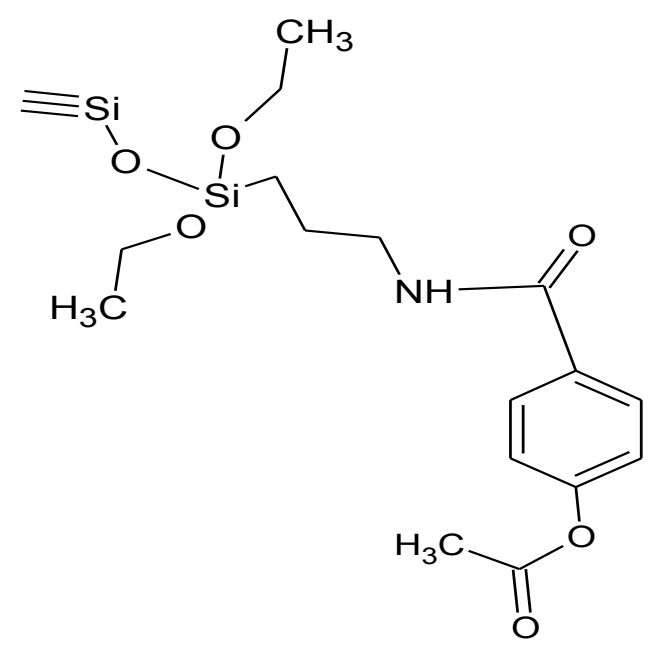

$\mathrm{SiO}_{2}$-acetylsalicylic acid nanoparticles<smiles>C#[Si]O[Si](CCCN=Cc1ccc(N(C)C)cc1)(OCC)OCC</smiles>

$\mathrm{SiO}_{2}$-p-dimethylaminobenzaldehyde nanoparticle

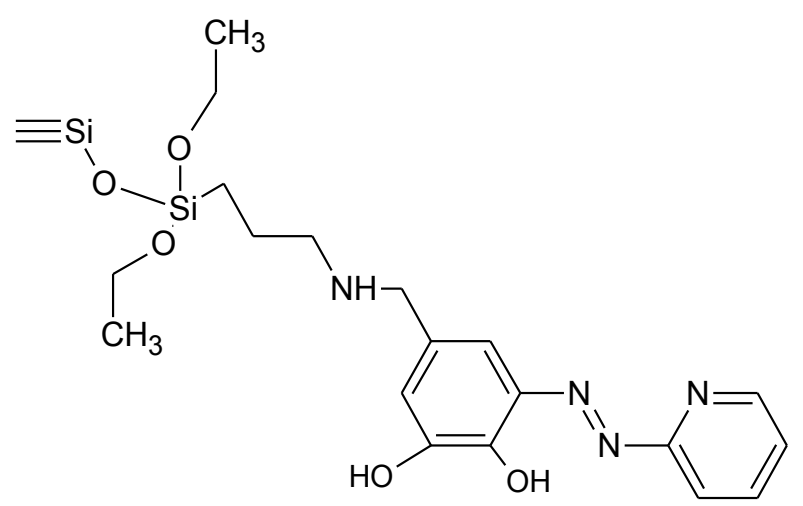

$\mathrm{SiO}_{2}$-4-(2-pyridylazo)-resorcinol nanoparticle

Fig 1. Chemical modification of silica nanoparticles with various silanes and ligands. 


\section{FULLERENES AND CARBON NANOTUBES}

Buckminster fullerenes, third allotropic form of carbon, are closed-cage carbon molecules containing pentagonal and hexagonal rings arranged in such a way that they have the formula $\mathrm{C} 20 \mathrm{pm}$, with $\mathrm{m}$ being an integer number. Fullerenes comprise a wide range of isomers and homologous series, from the most studied $\mathrm{C} 60$ or $\mathrm{C} 70$, to the so-called higher fullerenes, C240, C540, and C720. The analytical potential of C60 as an effective sorbent material for preconcentration of metals was recently demonstrated. Subsequent experiments with $\mathrm{C} 60$ and $\mathrm{C} 70$ fullerenes showed that both sorbents have a high analytical potential for metal preconcentration probably because of their large molecular surface area and volume. Higher sensitivity and selectivity are obtained with neutral chelates than by formation of ion pairs. Fullerenes with chemically modified surfaces combine the advantages of high enrichment factors offered by the large surface area and the selectivity associated with impregnated chemical groups. For example, C60 can be covalently bonded to classical chelating reagents, leading to stable fullerene derivatives that join the advantages of both compounds.

Since its discovery in 1991,carbon nanotubes (CNTs) have attracted great attention because of their unique properties. CNTs can be visualized as a sheet of graphite that has been rolled into a tube, and are divided into single-walled carbon nanotubes (SWNTs) and multi-walled carbon nanotubes (MWNTs) according to the carbon atom layers in the wall of the nanotubes. With the great progress in the methods of preparing CNTs, large efforts have been devoted to their fields of application. Their perceived novel mechanical and electronic properties, large specific surface area and high thermal stability indicate their tremendous potential for engineering applications, such as hydrogen storage, field emission, quantum nanowires, catalyst supports, chemical sensors and packing material for gas chromatography. The hexagonal arrays of carbon atoms in graphite sheets of CNTs surface have a strong interaction with other molecules or atoms, which make CNTs a promising adsorbent material substituted for activated carbon in many ways.

Table 1. Some examples of nanomaterials used as nanoadsorbents for toxins/pollutants.

\begin{tabular}{|c|c|c|c|c|c|}
\hline Nanomaterial & Modification & Analyte & Sample & Instrument & Ref \\
\hline $\mathrm{Fe}_{3} \mathrm{O}_{4}$ & Dithizone & $\mathrm{Cr}, \mathrm{Cu}, \mathrm{Pb}, \mathrm{Zn}$ & Environmental & ICP-OES & 1 \\
\hline $\mathrm{Fe}_{3} \mathrm{O}_{4} / \mathrm{TiO}_{2}$ & $\begin{array}{l}\text { Malachite green } \\
\text { carbinol }\end{array}$ & $\mathrm{Cd}, \mathrm{Cr}, \mathrm{Mn}, \mathrm{Cu}$ & Environmental & ICP-MS & 2 \\
\hline $\mathrm{Fe}_{3} \mathrm{O}_{4}$ & Zincon & $\mathrm{Pb}$ & $\begin{array}{l}\text { Natural drinking } \\
\text { water }\end{array}$ & FAAS & 3 \\
\hline $\mathrm{Fe}_{3} \mathrm{O}_{4} / \mathrm{SiO}_{2}$ & Schiff base & $\mathrm{Pb}, \mathrm{Cd}, \mathrm{Cu}$ & $\begin{array}{l}\text { Food, biological, } \\
\text { water samples }\end{array}$ & AAS & 4 \\
\hline $\mathrm{Fe}_{3} \mathrm{O}_{4}$ & $\begin{array}{c}\text { 3-(trimethyl)-1 } \\
\text {-propantiol } \\
\text { 2-amino-5-mercaptc } \\
\text { 1,3,4-thiadiazole }\end{array}$ & Ag, $\mathrm{Cd}, \mathrm{Cu}, \mathrm{Zn}$ & Environmental & ICP-OES & 5 \\
\hline $\mathrm{Fe}_{3} \mathrm{O}_{4}$ & Bismuthiol-II & $\mathrm{Cr}, \mathrm{Cu}, \mathrm{Pb}$ & Environmental & ICP-OES & 6 \\
\hline $\mathrm{Fe}_{3} \mathrm{O}_{4}$ & MIL-101 & PAHs & Environmental & ICP-OES & 7 \\
\hline $\begin{array}{c}\mathrm{Fe}_{3} \mathrm{O}_{4} \\
\text { /barium algina }\end{array}$ & -.-- & PAHs, Phthalate ester & Water & ICP-OES & 8 \\
\hline
\end{tabular}




\begin{tabular}{|c|c|c|c|c|c|}
\hline $\mathrm{Fe}_{3} \mathrm{O}_{4}$ & $\begin{array}{l}\text { Poly(divinylbenzene- } \\
\text { co-methacrylic acid) }\end{array}$ & EDCs & Water & LC-MS & 9 \\
\hline $\mathrm{Fe}_{3} \mathrm{O}_{4}$ & Alkyl carboxylate & PAHs & Water & HPLC & 10 \\
\hline $\mathrm{Fe}_{3} \mathrm{O}_{4}$ & $-{ }_{-1}$ & PAHs & Soil & HPLC & 11 \\
\hline $\mathrm{Fe}_{3} \mathrm{O}_{4}$ & Graphene & Triazole fungicide & water & NA & 12 \\
\hline $\mathrm{Fe}_{3} \mathrm{O}_{4}$ & Pyridine & $\mathrm{Pb}, \mathrm{Cd}$ & Tree leaf & FAAS & 13 \\
\hline MWCNs & & PAHs & Water & NA & 14 \\
\hline MWCNs & ----- & PAHs & Water & GC-MS & 15 \\
\hline $\mathrm{Al}_{2} \mathrm{O}_{3}$ & ------ & $\mathrm{Cd}, \mathrm{Pb}$ & Water, herbal & FAAS & 16 \\
\hline $\mathrm{AgO}$ & ------ & Pyrene & Water & NA & 17 \\
\hline AuNPs & ------ & EDCs & Water, real & NA & 18 \\
\hline AuNPs & ----- & Aflatoxin B1 & Food & HPLC & 19 \\
\hline AuNPs/Silica & ----- & $\mathrm{Hg}$ & Water & AFS & 20 \\
\hline AuNPs & ------ & $\begin{array}{l}\text { chlorpyrifos, } \\
\text { Malathion }\end{array}$ & $\begin{array}{l}\text { Surface } \\
\text { water }\end{array}$ & NA & 21 \\
\hline $\mathrm{AuNPs} / \mathrm{Al}_{2} \mathrm{O}_{3}$ & & $\begin{array}{l}\mathrm{MeHg}^{+} \\
\mathrm{EtHg}^{+}\end{array}$ & Water & ICP-MS & 22 \\
\hline AuNPs & Rhodamine $6 \mathrm{G}$ & $\mathrm{Hg}$ & $\begin{array}{c}\text { Water } \\
\text { Fish }\end{array}$ & AFS & 23 \\
\hline AuNPs & ----- & $\mathrm{Hg}, \mathrm{Pb}, \mathrm{Cu}$ & Water & NA & 24 \\
\hline $\mathrm{SiO}_{2}$ & PAN & $\mathrm{Zn}, \mathrm{Mn}$ & Food, water & SP & 25 \\
\hline $\mathrm{SiO}_{2}$ & PAN & $\mathrm{Sb}$ & Water & SP & 26 \\
\hline $\mathrm{SiO}_{2}$ & DHAQ & $\mathrm{Pb}$ & Water & SP & 27 \\
\hline $\mathrm{SiO}_{2}$ & RATP & $\mathrm{Cd}$ & Food, water & SP & 28 \\
\hline $\mathrm{SiO}_{2}$ & DHAQ & $\mathrm{Cd}$ & Water & SP & 29 \\
\hline $\mathrm{SiO}_{2}$ & PAN & Zineb, Maneb & Water & SP & 30 \\
\hline $\mathrm{SiO}_{2}$ & RATP & Co & Food, water & SP & 31 \\
\hline $\mathrm{SiO}_{2}$ & RATP & $\mathrm{Cu}$ & Vegetables & SP & 32 \\
\hline
\end{tabular}

CNTs possess a high electrical conductivity, chemical stability, mechanical strength, large specific surface area, and high thermal stability, indicating potential for various applications and one of the applications of the CNTs is as adsorbent material: the hexagonal arrays of carbon atoms in graphite sheets of CNTs surface have a strong interaction with other molecules or atoms, revealing a great analytical potential as a solid-phase extraction adsorbent. Additionally, CNTs' surfaces can be modified by introducing various organic functional groups, thus providing a strongly physic sorbing surface area, adjustable surface charge, and a source of protons for chemical ionization. It has been demonstrated that the surfaces of CNTs can be easily modified in numerous ways, either by covalent or noncovalent functionalization. All the facts mentioned before reveal that carbon nanotubes, and specially multiwalled carbon nanotubes, have great analytical potential as an effective solid-phase extraction adsorbent for chelates or ion pairs of metal ions, organic compounds, and organometallic compounds. 


\section{OTHER MATERIALS FOR NANOREMEDIATION}

Biosensors and affinity sensor devices have been shown to have the ability to provide rapid, cost effective, specific and reliable quantitative and qualitative analysis. To date the developments in nanomaterials and biosensor fabrications technology is moving rapidly with new and novel nanobiorecognition materials being developed which can be applied as the sensing receptors for mycotoxins analysis. Biosensors, as tools have proofed to be able to provide rapid, sensitive, robust and cost effective quantitative methods for on-site testing. Developing biosensor devices for different mycotoxins are attracting much research interest in recent years with a range of devices are being developed and reported in the scientific literature. However, with the advent of nanotechnology and its impact on developing ultrasensitive devices, mycotoxins analysis is benefiting also from the advances taking place in applying nanomaterials in sensors development. The application of nanotechnology in biosensors can range from the transducer device, the recognition ligand, the label and the running systems (e.g. instruments). Their application in sensor development has been due to the excellent advantages offered by these materials in miniaturisation of the devices, signal enhancements which result in high precision and accuracy and also amplification of signal by the use of nanoparticles as labels. The high surface to volume ratio offered by nanomaterials makes these devices very sensitive and can allow a single molecule detection which is very attractive in contaminant monitoring such as toxins. The development of micro/nanosensor devices for toxins analysis is increasing due to their extremely attractive characteristics for this application.

The development of micro/nanosensor devices for toxins analysis is increasing due to their extremely attractive characteristics for this application. In principle these devices are miniature transducers fabricated using conventional thin and thick film technology. Their novel electron transport properties make them highly sensitive for low levels detection. Multi toxins detection (e.g Mycotoxins) in foods can be conducted using single micro/ nanoelectrode array chip with high sensitivity and rapid analysis time. The use of micro/nanoarrays for analysis applications in foods can produce highly sensitive sensors. Multi mycotoxins detection has also been reported in the literature using different sensor platforms combined with multi ELISA assays. Therefore, multi toxins can be detected on a single microelectrode array chip with multi-array working electrode, where different antibody is immobilised to detect a specific mycotoxin. Micro/nanoelectrodes arrays have unique properties which include; small capacitive charging current and faster diffusion of electro active species which will result in an improved response time and greater sensitivity. The use of lab-on-a-chip is expanding in all areas of analysis due to the advantages of using small samples to analyse several markers/ toxins, i.e offer high throughput analysis. These types of devices will be attractive for mycotoxin analysis since several toxins may exist in the same food or feed sample.

A range of sensors are being developed for mycotoxins based on the above technologies which can be applied in the farm or the factory and operated by unskilled personnel. Current trends to produce chip-based micro/nanoarrays for multi mycotoxins analysis are challenging but possible and it will have significant impact on risk assessment testing. The use of nanoparticles such as gold, silver, metal oxides and quantum dots assay developments will enhance the capability of the biosensor technology for mycotoxins analysis. Early and sensitive detection will aid in eliminating these toxins from entering the food chain and preventing ill health and protecting life. 
The development of biosensors for the rapid, reliable and low-cost determination of mycotoxins in foodstuffs has received considerable attention in recent years, and various types of assays have already been devised for several of the major groups of mycotoxins. One format uses the phenomenon of surface plasmon resonance (SPR) to detect the change in mass that occurs when mycotoxin-specific antibodies attach to a mycotoxin that has been covalently bonded to the surface of a sensor chip. A recent application developed and optimized for measuring deoxynivalenol in wheat extracts gave results that were in good agreement with LC-MS data. Moreover, SPR sensor chips with immobilized deoxynivalenol could be re-used more than 500 times without significant loss of activity. Because the instrumentation is now commercially available, this format could find widespread application to future mycotoxin analysis. A second format using fibre-optic probes can be adapted for continuous monitoring of mycotoxin levels. This sensor uses the evanescent wave of light that can form around the surface of an optical fibre. Antibodies attached to the surface of the fibre trap fluorescent mycotoxins (e.g. aflatoxins) or fluorescent analogues of mycotoxins (e.g., derivatized fumonisins) with the evanescent zone, permitting their detection. Two different bench top devices have been designed for the fumonisins and aflatoxins. Unfortunately, most of the SPR and fibre optic biosensor procedures for mycotoxin analysis still require some form of sample clean-up/ preconcentration in order to be truly effective in the analysis of real samples and to achieve adequate sensitivity. Moreover, the majority of these devices lack the ability to perform simultaneous analyses of multiple samples. Recently, array biosensors have been developed and demonstrated for a variety of applications. The ability of arraybiosensors to analyse multiple samples simultaneously for multiple analytes offers a significant advantage over other types of biosensors. In particular, a rapid, multianalyte array biosensor developed by Ngundi et al. at the Naval Research Laboratory of Washington D.C., USA, has demonstrated the potential to be used as a screening and monitoring device for clinical, food and environmental samples. The device, which is portable and fully automated, can be used with different immunoassay formats.One interesting application is the development of a competitive immunoassay for the detection and quantification of ochratoxin $\mathrm{A}$ in a variety of spiked food and beverage samples. A simple extraction procedure was employed with no need for clean-up or preconcentration of the sample extract. This is the first demonstration that a rapid biosensor can be used in a competitive assay format to detect a mycotoxin in extracts of relevant foods. However, further work aimed at developing a dual-analyte assay for deoxynivalenol and ochratoxin A showed that improvements are still necessary to reduce the analysis time and increase the sensitivity.

Carbon nanotubes were used for the determination of zearalenone in urine samples by F. Andres et al. Multi-walled carbon nanotubes modified with an enzyme, aflatoxin detoxifizyme. MWCNTs were used for enzyme immobilization aflatoxins detoxifizyem (APTZ) and for the determination of sterigmatocystin and also carbon nanotubes field effect transistors (FET) that had been functionalized with protein G and IgG to detect Aspergillus flavus in contaminated milled rice. Optical sensors based on nanomaterials have been applied much less to the detection of analytes of interest in the food industry. Quantum dots (QD) are practically the only nano material. QD are nanocrystals of inorganic semiconductors that are somewhat restricted to a spherical shape of around 2 to $8 \mathrm{~nm}$ diameter. Their fluorescent properties are size-dependent and, therefore, they can be tuned to emit at desired wavelengths (between 400 and $2000 \mathrm{~nm}$ ) if synthesized in different composition and size. In this way, QDs of different sizes can be excited with a single wavelength and emission controlled at different wavelengths, thus providing for 
simultaneous detection. This, together with their highly robust emission properties, make them more advantageous for labelling and optical detection than conventional organic dyes. Their high quantum yields and their narrow emission bands produce sharper colours, lead to higher sensitivity and the possibility of multiplexing of analysis. Costa et al. have reviewed the progress in exploiting these novel probes in optical sensing, as well as their still unexploited sensing capabilities. In the analytical chemistry field their major application has been as fluorescent labels while an application to food analysis is, up to now, unexploited. Goldman et al. have used QDs for fluoroimmunoassays of toxins. They detected four toxins simultaneously, three of which are naturally responsible for food or water-borne sickness. The CdSe-ZnS core-shell QDs were capped with dihydrolipoic acid and bioconjugates with the appropriate antibodies were prepared. A sandwich immunoassay was performed in microtitre plates where the toxins and different QDs were incubated for an hour. Fluorescence was measured at adequate wavelengths and, although there was spectral overlap, deconvolution of spectra revealed fluorescence contribution of all toxins. Signals increased with toxin concentration in different ranges according to the particular toxin. No LODs were reported. Although authors treated the bioconjugate QDs as fluororeagents, they can be considered as "chemosensing devices". Ultrasensitive densitometry detection of cytokines was with nanoparticle modified aptamers. Gold nanoparticles were also employed for an immunoassay for the detection of aflatoxin B1 (AFB1) in foods.

\section{CONCLUSION}

Decontamination is the reduction or removal of chemical and biological agents by means of physical, chemical neutralization or detoxication techniques. Nanotechnology has shown huge potential in areas as diverse as drug development, water decontamination, information and communication technologies, and the production of stronger, lighter materials and human health care. Water and air are two vital components of life on earth; the existence of life on earth is made possible largely because of their importance to metabolic processes within body. Clean and fresh water and air are essential for the existence for life. The recent development of nanotechnology has raised the possibility of environmental decontamination through several nanomaterials, processes and tools. This review summarizes the expertise of various approaches of decontamination for successful realization of remediation in environment.

\section{References}

[1] He.et al., Talanta 88 (2012) 507-515.

[2] Zhang et al., Talanta 94 (2012) 278-283.

[3] Jiang et al., Talanta 94 (2012) 251-256.

[4] Khoshar et al., Talanta 97 (2012) 87-95.

[5] Mashhadizadeh et.al., J. Hazard. Mater. 190 (2011) 1023-1029.

[6] Suleiman et al., Talanta 77 (2009) 1579-1583.

[7] Huo et al., Analyst 137 (2012) 3445-3451. 
[8] Lu et. al., Anal. Chim. Acta. (2010) 665, DOI:10.1016/j.aca2010.03.026.

[9] Li. et al., J. Chromatogr A 1217 (2010) 1219-1226.

[10] Gomez et.al., Anal. Chem. 81 (2009) 9012-9021.

[11] Yan et.al., J. Sep. Sci. 34 (2011) 716-723.

[12] Xie et. al., J. Sep. Sci. 35 (2012) 2266-2272.

[13] Sayar et.al., Bio. Trace. Elem. Res. 150 (2012) 403-410.

[14] Guo et al., J. Chromatogr A. 1218 (2011) 9321-9327.

[15] Wang et al., J. Chromatogr A. 1173 (2007) 27-36.

[16] Ezoddin et al., J. Hazard. Mater.178 (2010) 900-905.

[17] Vasileva et al., J. Anal. Chem. 64 (2009) 1214-1216.

[18] Noh et. al., Electrophoresis 31(18) (2010) 3053-3060.

[19] Xiulan et.al., Intern. J. food microbiol. 99 (2005) 185-194.

[20] Leopold et.al., Anal. Chem. 81 (2009) 3421-3428.

[21] Lisha et.al., J. Environ Sci. Health B 44 (2009) 697-705.

[22] Si et al., Environ. Sci. Technol. 46 (2012) 2724-2730.

[23] Chang et.al., Environ Sci. Technol. 45 (2011) 1534-1539.

[24] Lin et al., Analyst 136 (2011) 863-871.

[25] Anupreet et. al., Eurasian J. Anal. Chem. 4 (2009) 234-244.

[26] Anupreet et al., J. AOAC Inter. 93 (2010) 1302-1307.

[27] Anupreet et. al., EJEAFChe. 9 (2010) 752-759.

[28] Anupreet et al., EJEAFChe. 9 (2010) 1334-1342.

[29] Anupreet et al., J. Chilean Chem. Soc. 55 (2010) 431-434.

[30] Anupreet et al., Eurasian J. Anal. Chem. 6 (2011) 1-9.

[31] Anupreet et. al., Sep Sci. 3 (2011) 1-7.

[32] Anupreet et al., Advances in nanoparticles 1 (2012) 1-7. 